\title{
Author Index Vol. 58, 1996
}

Adamopoulos, G. 78 Albers, F.W.J. 271 Al-Muhaimeed, H. 74 Amedee, R.G. 27 Anniko, M. 136,280 Antolí-Candela, F. 23 Antoniades, K. 347 Aoyagi, M. 175 Apostolopoulos, N. 78 Arnold, W. 127, 164, 195,229 Aso, S. 233 Attallah, M.S. 36

Bartenstein, P. 195 Baumgartner, W. 127 Baur, S. 127 Bergenius, J. 277 Bergler,W. 219 Bergstedt,H. 333 Brantberg, K. 277 Bredbacka, S. 99 Buisseret, Th. 315 Bujía, J. 23 Bumber, Z. 55 Bura,M. 55

Caversaccio, M. 164 Chung, J.W. 51 Clement, P.A.R. 315 Comacchio, F. 295

Davies,W.E. 121 Dejonckere, P.H. 326 D’Eredità,R. 295 Deron,P. 167 DiLeo,M.D. 27

Dunnebier, E.A. 271

Egawa,M. 258 Ehrenberger, K. 121,127 Engström, C.-F. 105

Fede,A. 253 Felix, D. 121 Felix, H. 121 Ferekidis, E. 78 Flisberg,K. 39 Francini, F. 253

Friedmann, I. 182 Fujimori, I. 110, 147 Fukuda,H. 171 Furuta, S. 258

Furuya,N. 238 Fuse, T. 175

Ganesan, S. 178 Ganzer,U. 248 Gitter,A.H. 1 Godbersen, G.S. 115 Goossens, A. 167 Gordts,F. 315 Goto,R. 147 Gstöttner,W. 127 Guyot,J.-P. 304 Guzman Tapia, M. 23 Gysin, C. 306 Haas, I. 248 Halama,A.R. 167 Harada, N. 320 Haraldsson, P.-O. 99 Harar,R.P.S. 178 Hatt,H. 183 Häusler,R. 87 Heiniger-Bürki, Ch. 121 Helböck,C. 213 Helms, J. 127 Hentona, H. 224 Herdegen, T. 262 Himi,T. 298 Hirota, R. 258 Hisamatsu, K.-i. 110, 147 Holly, A. 23 Honjo,I. 200 Hörmann, K. 219 Hülse,M. 219 Hultcrantz, M. 333 Hydén,D. 338

Igarashi,M. 204,301 Iida,M. 204 Ikeda,H. 320 Ilberg, C. von 127 Imamura, S.-I. 110 Imanaka, M. 93 Imboden,H. 121 Inamura, H. 175 Ishida,K. 204 Ishii,M. 301 Itoh,T. 93 Iwasaki, S. 343 Jinnouchi, K. 4 Juncker, C. 219

Kaga,K. 343 Kakoi,H. 136,280 Kano,Y. 288 Kanonier, G. 213 Kanzaki, J. 32 Karakasis, D. 347 Kastenbauer, E. 23 Kataura,A. 298 Kato,A. 9 Kawaida, M. 171 Keilmann, A. 262 Kiefer,J. 127 Kikushima, K. 147 Kim,Y.-J. 82 Kimura, H. 233 Kitamura, K. 42 Klapan, I. 55 Klimek,L. 46,157 Kobayashi,T. 301 Kohno,N. 171 Kojima,Y. 238 Koldovsky,P. 248 Komatsuzaki, A. 224 Kontrogianni, A. 78 Korves, B. 46 Kubo,N. 320 Kudo,Y. 208 Kühn,A.G. 229 Kunihiro, T. 32 Kuroda,M. 343

Landgrebe, M. 266 Laskawi, R. 13,266 Lebacq, J. 326 Lengersdorf, A. 157 Lippert, B.M. 115 Lise,M. 253 Lüttges, J. 115

MacDonald, A. 178 Marchiori, C. 253, 295 Masutani, H. 9 Mendel, L. 333 Min,Y.-G. 51,82

Miura, M. 200 Miyata, M. 42 Moriyama, H. 301 Mösges, R. 46, 157 Müller,J. 127 Murakami, Y. 110,147

351

Naito,Y. 200 Naitoh,A. 204 Nakagawa, H. 171 Nakai,Y. 9

Nakamura,A. 171,320 Nakamura, T. 175 Nakamura, Y. 238 Nakayama, H. 110 Nathanson, A. 105 Nishida,I. 68 Nishimoto, K. 258 Nomura, K. 204 Nomura, Y. 208 Nozawa, I. 110 Ntouniadakis, E. 78

Odawara,Y. 298 Oestreicher, E. 195 Ohashi,N. 233 
Pawankar, R. 4 Peschel,O. 164 Petersen, H. 39 Petroianu, G. 219 Pfennigdorff, Th. 127

Pilati,P.L. 253 Prior, A.J. 178 Psarommatis, I. 78 Psimopoulou, M. 347 Pucciarelli, S. 253

Pulle $\pi$,D. 178

Räisänen, S. 151 Rantala,H. 151 Römer,W. 195

Sakai,M. 204 Sakata, H. 238 Sakata, M. 298 Sakurai, H. 288 Satoh,A. 32 Satoh,J.-i. 298

Satoh,Y. 32 Sawaishi, M. 298 Schickinger, B. 121 Schön,F. 127 Schrott-Fischer, A. 213

Schultze, V. 157 Schwaiger, M. 195 Schweri,T. 87 Segenhout, J.M. 271 Sekiguchi,C. 301

Seppey,M. 87 Shidara, J.-I. 288 Shiotani,A. 171 Simovic, S. 55 Skatvedt,O. 243 Sobin,J. 105

Spaeth, J. 157 Stenfors, L.-E. 151 Stephan, K. 127 Sudhoff,H. 23 Sugimura, H. 311 Sugiyama, T. 9 Suzuki, T. 175

Takahashi,H. 93,200 Takahashi, J. 42 Takumida, M. 68 Tanaka, H. 224 Tange,R.A. 143

Thumfart,W. 127 Thurner,K.H. 213 Toida,S. 288 Tomiyama, S.-i. 4 Toppan, P. 253 Tribukait, A. 277 Tschopp,K.P. 306

Ueda, H. 61 Ulmer,H. 213

Vahtsevanos, K. 347

Watanabe,Y. 233 Wennmo,C. 39 Werner, J.A. 115 Wimmer, M. 213 Wit,H.P. 271 Wolff, J.R. 13,266

Yagi,M. 343 Yamada,T. 147 Yamamoto,Y. 61,93 Yamashita,T. 320 Yamasoba, T. 311

Yanagita, N. 61 Yashiro,T. 301 Yasuda, H. 288 Yoshihara, S. 32 Yun,Y.-S. 82

Zakzouk, S.M. 74

352

Author Index Vol. 58, 1996 